\title{
Vibration suppression for strings with distributed loading using spatial cross-section modulation
}

Sorokin, Vladislav; Thomsen, Jon Juel

Published in:

Journal of Sound and Vibration

Link to article, DOI:

10.1016/j.jsv.2014.09.028

Publication date:

2015

Document Version

Peer reviewed version

Link back to DTU Orbit

Citation (APA):

Sorokin, V., \& Thomsen, J. J. (2015). Vibration suppression for strings with distributed loading using spatial cross-section modulation. Journal of Sound and Vibration, 335, 66-77. https://doi.org/10.1016/j.jsv.2014.09.028

\section{General rights}

Copyright and moral rights for the publications made accessible in the public portal are retained by the authors and/or other copyright owners and it is a condition of accessing publications that users recognise and abide by the legal requirements associated with these rights.

- Users may download and print one copy of any publication from the public portal for the purpose of private study or research.

- You may not further distribute the material or use it for any profit-making activity or commercial gain

- You may freely distribute the URL identifying the publication in the public portal

If you believe that this document breaches copyright please contact us providing details, and we will remove access to the work immediately and investigate your claim. 


\title{
Vibration suppression for strings with distributed loading using spatial cross-section modulation
}

\author{
Vladislav S. Sorokin ${ }^{1,2}$, Jon Juel Thomsen ${ }^{1}$ \\ ${ }^{1}$ Department of Mechanical Engineering. Technical University of Denmark \\ Nils Koppels Allé, Building 404, 2800 Kgs. Lyngby, Denmark \\ ${ }^{2}$ Institute of Problems in Mechanical Engineering RAS \\ V.O., Bolshoj pr. 61, St.Petersburg, 199178, Russia \\ vladsor@mek.dtu.dk \\ phone: +4550121813 \\ fax: +4545251961
}

\begin{abstract}
A problem of vibration suppression in any preassigned region of a bounded structure subjected to action of an external time-periodic load which is distributed over its domain is considered. A passive control is applied, in that continuous spatially periodic modulations of structural parameters are used as a means for vibration suppression. As an example, stationary vibrations of a string under action of a distributed time-periodic load are studied. This system in a simplified form models such processes as interaction between membranes and colloids, oscillations of transmission lines under action of rain and wind, and dynamics of suspension bridges and stay cables. Suppression of vibration in predefined regions of the string is performed by continuous spatial modulation of its cross-section.

For analyzing the problem considered a novel approach named the method of varying amplitudes is employed. This approach is applicable for solving differential equations without a small parameter, and may be considered as a natural continuation of the classical methods of harmonic balance and averaging. As a result, optimal parameters for the string cross-sectional area modulation are determined for the cases of harmonically, uniformly and arbitrarily distributed load, which allows for completely suppressing or considerably reducing vibration in the prescribed part of the string (compared to the case without modulation).
\end{abstract}

vibration suppression; bounded structure; distributed loading; continuous spatially periodic modulation; the method of varying amplitudes.

\section{Introduction}

The paper is concerned with a problem of vibration suppression in any preassigned region of a bounded structure subjected to action of an external time-periodic load which is distributed over its domain. Similar problems of sound and vibration isolation gained much attention in the recent years (e.g. [1-8]). The approaches proposed for treating such problems may be divided in two groups: active control, when structural parameters are varied in time [3, 8-12], and passive control, when these parameters are changing only in space [2, 5, 7, 13-15]. In most cases behavior is considered in presence of a point load or an excitation source, and boundary conditions are not taken into account [2-6, 12-14, 16-17]. Then the problem reduces to identifying frequency stop and pass bands, and their subsequent tailoring by spatial/temporal modulations of system parameters. In the present paper the problem of vibration suppression is considered in a different formulation: behaviour of a bounded structure under action of a distributed load is studied. A passive control is applied, in that continuous spatially periodic modulations of structural parameters are proposed to be used as a means for vibration suppression. We note that the problem under study is closely related to 
those solved by the method of topology optimization [5], which is a popular method for obtaining an optimal layout of one or several material constituents in structures and materials. However, the important advantage of the analytical approach employed in the present paper over this and other numerical methods is the ability to provide insight into, e.g., explicit dependencies of structural dynamic characteristics on parameters.

As an illustrative example, consider vibrations of a string under action of an external distributed time-periodic load. Suppression of vibration in predefined regions of the string is performed by continuous spatial modulation of its cross-section. This simple system is a generic model for various relevant problems, e.g. interaction between membranes and colloids in biochemistry [18], oscillations of transmission lines under action of rain and wind [19], and dynamics of suspension bridges and stay cables [20]. Moreover, it serves to reveal general effects of continuous spatially periodic modulations on oscillations of bounded structures subjected to distributed loading, and illustrates possible advantages and disadvantages of the proposed technique of vibration suppression.

For analyzing the problem considered a novel approach named the method of varying amplitudes (MVA) is employed. This approach is inspired by the method of direct separation of motions (MDSM) [21, 22], and may be considered a natural continuation of the classical methods of harmonic balance [23] and averaging [24-26]. It implies representing a solution in the form of harmonic series with varying amplitudes, but in contrast to the averaging methods the amplitudes are not required to vary slowly. Thus the MVA does not assume the presence of a small parameter in the governing equations, or any restrictions on the sought solution. This, in particular, makes it convenient for analysis of the considered problem which implies solving differential equations without a small parameter.

The paper is structured as follows: In Section 2 the equations of motion for the inhomogeneous string are presented, and the specific aims of the analysis are outlined. Section 3 is concerned with the case of spatially harmonic external load: In Section 3.1 a solution of the governing equations by the MVA is provided, and essence of the method is briefly described. In Section 3.2 optimal parameters of the string cross-sectional area modulation are determined that ensures complete suppression or considerable reduction of vibration in predefined regions of the string. Section 4 is concerned with the case of spatially uniform external load. In Section 4.1 the corresponding equations are solved by the MVA, while Section 4.2 calculates optimal parameters of the string cross-sectional area modulation. Finally, Section 5 is concerned with the case of arbitrarily distributed external load.

\section{Governing equations}

Consider vibrations of a string with variable cross-section under action of a distributed load which are described by the equation

$$
\rho S(x) \frac{\partial^{2} u}{\partial t^{2}}-T \frac{\partial^{2} u}{\partial x^{2}}=f(x, t),
$$

Where $\rho$ is density of the string material, $T$ the tension force (assumed constant), $u(x, t)$ the lateral deflection of the string, $x \in[0 ; l]$ the axial coordinate, $S(x)$ the variable cross-sectional area, and $f(x, t)$ the external load per unit length. The boundary conditions are: $u(0, t)=u(l, t)=0$, and $l$ is length of the string; motion of the string is studied at zero initial conditions. Dissipation is assumed to be negligibly small. The case of a time-varying load of period $\Theta$ is considered:

$$
f(x, t)=f(x, t+\Theta) .
$$

This is justified and common for most of the abovementioned problems; e.g. impact of rain and wind on transmission lines and stay cables [19, 20], and colloids on membranes [18] is usually modeled as time-periodic. Expanding $f$ in a trigonometric Fourier series gives: 


$$
f(x, t)=c_{0}(x)+\sum_{n=1}^{\infty}\left(c_{n}(x) \cos \left(\frac{2 \pi}{\Theta} n t\right)+d_{n}(x) \sin \left(\frac{2 \pi}{\Theta} n t\right)\right) .
$$

Consequently, since (1) is linear, the problem reduces to analyzing the case

$$
f(x, t)=f_{0}(x) \cos (\omega t+\phi),
$$

where $\omega=\frac{2 \pi}{\Theta} n$, and $\phi=0, \frac{\pi}{2}$. In this case the solution of (1) may be written in the form

$$
u(x, t)=A(x) \cos (\omega t+\phi)+w(x, t),
$$

where $w(x, t)$ is solution of the homogeneous equation corresponding to (1) at the same boundary conditions and the initial conditions: $w(x, 0)=-A(x) \cos \phi, \dot{w}(x, 0)=A(x) \omega \sin \phi$. Thus, the problem of minimizing the string response $u(x, t)$ turns to determining $A(x)$, and subsequent tailoring of $A(x)$ by cross-sectional area modulation. For $A(x)$ the following equation is composed, by inserting (3)-(4) into (1):

$$
\frac{d^{2} A}{d x^{2}}+\frac{\omega^{2} \rho S(x)}{T} A=-\frac{f_{0}(x)}{T}
$$

The aim is to determine modulations of the string cross-sectional area that would enable vibration suppression in predefined string regions for an arbitrarily distributed external load. To accomplish this we first expand $f_{0}$ in a trigonometric Fourier series:

$$
f_{0}(x)=a_{0}+\sum_{n=1}^{\infty}\left(a_{n} \cos \frac{2 \pi}{l} n x+b_{n} \sin \frac{2 \pi}{l} n x\right),
$$

and determine optimal cross-section modulation $S(x)$ for 1 ) the case of a spatially harmonic external load, and 2) the case of a spatially uniform external load. The sought modulation for an arbitrarily distributed load $f_{0}(x)$ is proposed to be approximated as the one corresponding to dominating spatial harmonic or as a certain superposition of the determined modulations.

\section{Spatially harmonic external load}

Consider the case of a spatially harmonic external load:

$$
f_{0}(x)=F_{0} \cos (k x+\varphi),
$$

where $k=\frac{2 \pi}{l} n, n$ is integer, and the phase $\varphi$ is arbitrary, so that various load distributions are captured. A modulation of the cross-sectional area $S(x)$ should be determined such that vibration in any predefined region of the string, e.g. near a certain point $x_{p}$, would be minimal. The well-known effect of parametric attenuation (e.g. [27]) is proposed to be employed for this purpose. Thus the following approximation to the sought modulation is used:

$$
S(x)=S_{0}\left(1+\chi \cos \left(2 k x+\theta_{n}\right)\right)
$$

where $\chi \in[0 ; 1)$ and $\theta_{n}$ are unknown parameters to be determined. Inserting (7) and (8) into (5) one obtains

$$
A_{1}^{\prime \prime}\left(x_{1}\right)+\delta\left(1+\chi \cos \left(2 x_{1}+\theta\right)\right) A_{1}\left(x_{1}\right)=\cos x_{1},
$$

where $x_{1}=k x+\varphi, \theta=\theta_{n}-2 \varphi, \delta=\omega^{2} \rho S_{0} / T k^{2}$ and $A_{1}\left(x_{1}\right)=-\left(T k^{2} / F_{0}\right) A\left(x_{1}\right)$, and primes denote derivatives with respect to $x_{1}$. The boundary conditions for $A_{1}\left(x_{1}\right)$ take the form:

$$
A_{1}(\varphi)=A_{1}(k l+\varphi)=0,
$$

since $x=0$ and $x=l$ correspond to $x_{1}=\varphi$ and $x_{1}=k l+\varphi$. Our aim is to study (9) in the widest possible range of external excitation parameters $\omega$ and $k$, so that parameter $\delta$ may 
take any value. However, consideration of such a general case, though possible, leads to very complicated expressions, so here we assume $\delta=O(1)$ (which comprises also the case $\delta<<1$ ); the case of large $\delta$ may be analyzed similarly.

\subsection{Solution by the method of varying amplitudes}

To solve (9), which does not contain a small parameter since $\delta=O(1)$ and $0 \leq \chi<1$, we employ a novel approach here named the method of varying amplitudes (MVA). This approach is a development of two modified versions of the method of direct separation of motions (MDSM) [21,22]. The first was introduced in [28], where the analysis of free vibrations of a string with variable cross-section was carried out, and is useful for studying dynamics of spatially periodic structures. The second was proposed in [29] for solving equations without an explicit small parameter. However, the latter approach implies a certain restriction on the sought solutions: only those which are close to periodic can be determined. This manifests itself in introducing an artificial small parameter, and representation of the solution in the form of a harmonic series with slowly varying amplitudes. The MVA avoids this restriction in that no small parameter is used in the solution process, and amplitudes of the solution are not required to vary slowly. Thus, the method does not imply separation of variables on slow and fast scales (the main idea of the MDSM) and hence cannot be named as a modified MDSM. Avoiding the use of a small parameter significantly broadens the applicability range of the MVA in comparison with the conventional MDSM [21,22] and the classical asymptotic methods [24-26], in particular the multiple scales method [26]. E.g. the solution of equation (9), which will be obtained by it, should be valid in the whole range of $\delta$-values considered $\delta=O(1)$.

So, a solution of (9) is sought in the form of harmonic series with varying amplitudes:

$$
A_{1}\left(x_{1}\right)=B_{11}\left(x_{1}\right) \cos x_{1}+B_{12}\left(x_{1}\right) \sin x_{1}+B_{21}\left(x_{1}\right) \cos 3 x_{1}+B_{22}\left(x_{1}\right) \sin 3 x_{1}+\ldots \text {, }
$$

where the amplitudes $B_{j 1}\left(x_{1}\right), B_{j 2}\left(x_{1}\right), j=1,2, \ldots m$, are not required to vary slowly in comparison with $\cos x_{1}, \cos 3 x_{1}$ etc. The shift from the original dependent variable $A_{1}\left(x_{1}\right)$ to $2 m$ new variables $B_{j 1}\left(x_{1}\right), B_{j 2}\left(x_{1}\right)$ implies that $2 m$ equations for these variables are needed. This can be accomplished by introducing constraints on these variables in the form of $2 m-1$ additional equations. With the MVA the constraints are introduced in the following way: substitute (11) into (9), and require $2 m-1$ groups of terms to equal zero. The last $2 m$ th equation will include all the remaining terms of the original equation. These $2 m-1$ groups of terms are proposed to be the coefficients of the involved spatial harmonics $\cos x_{1}, \sin x_{1}$, $\cos 3 x_{1}$ etc. Taking into account only the written terms in (11), one obtains four equations for the amplitudes $B_{11}\left(x_{1}\right), B_{12}\left(x_{1}\right), B_{21}\left(x_{1}\right), B_{22}\left(x_{1}\right)$ :

$$
\begin{aligned}
& B_{11}^{\prime \prime}+2 B_{12}^{\prime}+(\delta-1) B_{11}+\frac{\delta \chi}{2}\left(\left(B_{11}+B_{21}\right) \cos \theta-\left(B_{12}+B_{22}\right) \sin \theta\right)=1, \\
& B_{12}^{\prime \prime}-2 B_{11}^{\prime}+(\delta-1) B_{12}-\frac{\delta \chi}{2}\left(\left(B_{12}-B_{22}\right) \cos \theta+\left(B_{11}-B_{21}\right) \sin \theta\right)=0 \\
& B_{21}^{\prime \prime}+6 B_{22}^{\prime}+(\delta-9) B_{21}+\frac{\delta \chi}{2}\left(B_{11} \cos \theta+B_{12} \sin \theta\right)=0, \\
& \left(B_{22}^{\prime \prime}-6 B_{21}^{\prime}+(\delta-9) B_{22}+\frac{\delta \chi}{2}\left(B_{12} \cos \theta-B_{11} \sin \theta\right)\right) \sin 3 x_{1} \\
& =-\frac{\delta \chi}{2}\left(B_{21} \cos \left(5 x_{1}+\theta\right)+B_{22} \sin \left(5 x_{1}+\theta\right)\right) .
\end{aligned}
$$


So, we have restated the original equation (9) in the form of four equations (12)-(15), with $B_{11}, B_{12}, B_{21}, B_{22}$ as the dependent variables instead of $A_{1}$; no approximations are involved so far.

Now we introduce approximations by neglecting the right-hand side of (15), so that:

$$
B_{22}^{\prime \prime}-6 B_{21}^{\prime}+(\delta-9) B_{22}+\frac{\delta \chi}{2}\left(B_{12} \cos \theta-B_{11} \sin \theta\right)=0 .
$$

This simplification is valid when $\delta \chi / 2<<|\delta-9|$, i.e. the right-hand side of (15) is small in comparison with the leading term. In the present paper the case $\delta=O(1), \chi \in[0 ; 1)$ is considered, so this condition holds true. When $\delta>>1$, more harmonics should be taken into account in series (11), so that it will be justified to neglect the right-hand side of the last $2 m$ th equation for amplitudes $B_{m 1}\left(x_{1}\right), B_{m 2}\left(x_{1}\right)$. As is seen, the approximation implied in the MVA is quite similar to the one of the classical method of harmonic balance [23], i.e. the influence of higher harmonics is neglected.

Note that the classical averaging methods [24,25] also imply a shift of variables from the original dependent variable to new variables, typically amplitude and phase. But the corresponding constraint on the new variables in common practice takes the form of the Van der Pol condition [24,25]. The constraint employed in the MVA is closer to the one used in the conventional MDSM [21,22]; this method also requires a certain group of terms in the initial equation, describing "fast motions" [21,22], to equal zero.

So, the original equation (9) for $A_{1}$ is replaced by four equations (12)-(14), (16) for the amplitudes $\mathbf{B}=\left\{\begin{array}{llll}B_{11} & B_{12} & B_{21} & B_{22}\end{array}\right\}^{\mathbf{T}}$. This system of equations has eight eigenvalues and corresponding eigenvectors. Its general solution may be written in the form:

$$
\mathbf{B}=\mathbf{B}_{0}+C_{1} \mathbf{B}_{1} \exp \lambda_{1} x_{1}+C_{2} \mathbf{B}_{2} \exp \lambda_{2} x_{1}+\ldots+C_{8} \mathbf{B}_{\mathbf{8}} \exp \lambda_{8} x_{1}
$$

Here $\mathbf{B}_{0}$ is a particular solution of (12)-(14), (16), $\mathbf{B}_{j}$ and $\lambda_{j}(j=1, \ldots, 8)$ are eigenvectors and corresponding eigenvalues, and $C_{1}, \ldots, C_{8}$ arbitrary constants. Consequently, returning to the original variable $A_{1}\left(x_{1}\right)$ using (11), obtain that it should depend on these constants

$$
A_{1}\left(x_{1}\right)=A_{1}\left(x_{1}, C_{1}, \ldots, C_{8}\right),
$$

When $\chi=0$, the right-hand side of equation (15) equals zero, so solution of (12)-(14), (16) will give us an exact solution $A_{1}\left(x_{1}\right)$ of the initial equation (9). The eigenvalues and eigenvectors of (12)-(14), (16) in this case are the following:

$$
\begin{gathered}
\lambda_{1-4}= \pm i(\sqrt{\delta} \pm 1), \lambda_{5-8}= \pm i(\sqrt{\delta} \pm 3) \\
\mathbf{B}_{1-4}=\left\{\begin{array}{lll}
1 \mp i & 0 & 0
\end{array}\right\}^{\mathbf{T}}, \mathbf{B}_{5-8}=\left\{\begin{array}{lll}
0 & 0 & 1
\end{array} i\right\}^{\mathbf{T}} .
\end{gathered}
$$

The particular solution of (12)-(14), (16) is $\mathbf{B}_{0}=\left\{\left(\begin{array}{llll}(\delta-1)^{-1} & 0 & 0 & 0\end{array}\right\}^{\mathbf{T}}\right.$. As is seen, for any values of constants $C_{1}, \ldots, C_{8}$ the corresponding expression for $A_{1}\left(x_{1}\right)$ is an exact solution of the initial equation (9). E.g. if $C_{1}=1 \quad\left(\lambda_{1}=i(\sqrt{\delta}+1), \quad \mathbf{B}_{1}=\left\{\begin{array}{lll}1-i & 0 & 0\end{array}\right\}^{\mathrm{T}}\right)$ and $C_{2}=C_{3}=\ldots=C_{8}=0$, one finds:

$$
A_{1}\left(x_{1}\right)=\left(\frac{1}{\delta-1}+\exp \left(i(\sqrt{\delta}+1) x_{1}\right)\right) \cos x_{1}-i \exp \left(i(\sqrt{\delta}+1) x_{1}\right) \sin x_{1}=\frac{1}{\delta-1} \cos x_{1}+\exp \left(i \sqrt{\delta} x_{1}\right)(21)
$$

On the other hand, if $C_{1}=C_{2}=\ldots=C_{7}=0$ and $C_{8}=1\left(\lambda_{8}=-i(\sqrt{\delta}-3), \mathbf{B}_{8}=\left\{\begin{array}{lll}0 & 0 & 1-i\end{array}\right\}^{\mathrm{T}}\right)$, we get: 


$$
A_{1}\left(x_{1}\right)=\frac{1}{\delta-1} \cos x_{1}+\exp \left(\lambda_{8} x_{1}\right) \cos 3 x_{1}-i \exp \left(\lambda_{8} x_{1}\right) \sin 3 x_{1}=\frac{1}{\delta-1} \cos x_{1}+\exp \left(-i \sqrt{\delta} x_{1}\right)
$$

So, expression (18) will be the solution of (9) for any values of constants $C_{1}, \ldots, C_{8}$. However, there are only two boundary conditions (10) to satisfy. Thus, we can force six of these constants to equal zero, and confine ourselves to determining only two eigenvalues of the system (12)-(14), (16), e.g. the lowest eigenvalues. This significantly simplifies the application procedure of the MVA and the resulting expressions.

When $\chi \neq 0$, the particular solution of (12)-(14), (16) takes the form $\mathbf{B}_{0}=\left\{\begin{array}{llll}B_{110} & B_{120} & B_{210} & B_{220}\end{array}\right\}^{\mathbf{T}}$ where

$$
\begin{aligned}
& B_{110}=-\frac{4(\delta-9)\left(-36+40 \delta+\delta^{2}\left(\chi^{2}-4\right)+2(\delta-9) \delta \chi \cos \theta\right)}{1296-2880 \delta+8 \delta^{3}\left(19 \chi^{2}-40\right)-4 \delta^{2}\left(99 \chi^{2}-472\right)+\delta^{4}\left(16-12 \chi^{2}+\chi^{4}\right)}, \\
& B_{120}=\frac{8(\delta-9)^{2} \delta \chi \sin \theta}{1296-2880 \delta+8 \delta^{3}\left(19 \chi^{2}-40\right)-4 \delta^{2}\left(99 \chi^{2}-472\right)+\delta^{4}\left(16-12 \chi^{2}+\chi^{4}\right)} .
\end{aligned}
$$

The corresponding values of $B_{210}$ and $B_{220}$ are much smaller than $B_{110}$ and $B_{120}$ at $\delta=O(1)$, and thus not given here. As follows from (23), the minimal value of the particular solution amplitude $B_{10}=\sqrt{B_{110}^{2}+B_{120}^{2}}$ will be achieved at $\theta=\pi$ if $\delta<1$, and at $\theta=0$ if $\delta>1$. So, with the assigned task of vibration suppression, and confining ourselves to considering only the first case $\delta<1$, we let $\theta=\pi$; the case $\delta>1$ can be studied in a similar way.

Now determine the lowest two eigenvalues of (12)-(14), (16). To simplify the resulting expressions we employ the approximate relations between amplitudes $B_{21}, B_{22}$ and $B_{11}, B_{12}$ which may be obtained from (14), (16):

$$
B_{21} \approx \frac{\delta \chi}{2(\delta-9)} B_{11}, B_{22} \approx \frac{\delta \chi}{2(\delta-9)} B_{12} .
$$

These approximations are justified by the fact that for eigenvectors corresponding to the lowest eigenvalues $\left(B_{21}, B_{22}\right)<<\left(B_{11}, B_{12}\right)$, and thus $B_{21}, B_{22}$ may be taken into account only approximately. Substituting (24) into (12)-(13) we get

$$
\begin{aligned}
& B_{11}^{\prime \prime}+2 B_{12}^{\prime}+\left(\delta-1-\frac{\delta \chi}{2}-\frac{\delta^{2} \chi^{2}}{4(\delta-9)}\right) B_{11}=1, \\
& B_{12}^{\prime \prime}-2 B_{11}^{\prime}+\left(\delta-1+\frac{\delta \chi}{2}-\frac{\delta^{2} \chi^{2}}{4(\delta-9)}\right) B_{12}=0
\end{aligned}
$$

Differentiating (25) with respect to $x_{1}$, and substituting $B_{11}^{\prime}$ from (26) we get:

$$
B_{12}^{\prime \prime \prime}+2\left(\delta+1-\frac{\delta^{2} \chi^{2}}{4(\delta-9)}\right) B_{12}^{\prime \prime}+\left[\left(\delta-1-\frac{\delta^{2} \chi^{2}}{4(\delta-9)}\right)^{2}-\left(\frac{\delta \chi}{2}\right)^{2}\right] B_{12}=0 \text {. }
$$

The eigenvalues of (27) are governed by

$$
\lambda_{1-4}^{2}=-\left(\delta+1-\frac{\delta^{2} \chi^{2}}{4(\delta-9)}\right) \pm \sqrt{4\left(\delta-\frac{\delta^{2} \chi^{2}}{4(\delta-9)}\right)+\left(\frac{\delta \chi}{2}\right)^{2}}
$$

Thus the lowest two eigenvalues of (12)-(14), (16) are defined by the following approximate relation:

$$
\lambda^{2}=-\left(\delta+1-\frac{\delta^{2} \chi^{2}}{4(\delta-9)}\right)+\sqrt{4\left(\delta-\frac{\delta^{2} \chi^{2}}{4(\delta-9)}\right)+\left(\frac{\delta \chi}{2}\right)^{2}} .
$$


The resulting expressions for the amplitudes $B_{11}$ and $B_{12}$ take the form

$$
\begin{aligned}
& B_{12}=C_{1} \sinh \lambda x_{1}+C_{2} \cosh \lambda x_{1}, \\
& B_{11}=\frac{1}{\delta-1-\frac{\delta \chi}{2}-\frac{\delta^{2} \chi^{2}}{4(\delta-9)}}-\frac{2 \lambda}{\delta-1-\frac{\delta \chi}{2}-\frac{\delta^{2} \chi^{2}}{4(\delta-9)}+\lambda^{2}}\left(C_{1} \cosh \lambda x_{1}+C_{2} \sinh \lambda x_{1}\right) .
\end{aligned}
$$

Note that $|\lambda|<<1$ when $(\delta-1)^{2} \approx(\delta \chi / 2)^{2}$, in which case $B_{11}, B_{12}, B_{21}, B_{22}$ vary slowly with respect to $x_{1}$.

To determine $C_{1}, C_{2}$ we employ the boundary conditions (10) for $A_{1}\left(x_{1}\right)$. The corresponding expressions for these constants are lengthy, and thus not given here. So, the solution $A_{1}\left(x_{1}\right)$ of the considered problem is composed in the form (11), where amplitudes $B_{11}, B_{12}$ are defined by (30), and amplitudes $B_{21}, B_{22}$ by (24).

\subsection{Vibration suppression in predefined regions of the string}

The stationary vibrations near a certain point $x_{p}$ of the string will be maximally suppressed if the amplitude $B_{1}=\sqrt{B_{11}^{2}+B_{12}^{2}}$ in this point is minimized, i.e. if

$$
\left.B_{1}\right|_{x=x_{p}}=B_{1}\left(k x_{p}+\varphi\right)=\sqrt{B_{11}^{2}\left(k x_{p}+\varphi\right)+B_{12}^{2}\left(k x_{p}+\varphi\right)} \rightarrow \text { min . }
$$

The size of the region near $x_{p}$ in which vibration is minimized depends on the rate of change of $B_{1}$ with respect to the spatial coordinate $x_{1}$. As noted above, $B_{1}$ varies slowly with $x_{1}$ for a broad range of system parameters, so the region will be wide in most cases.

The effect of cross-section modulation turns out to be most pronounced when $\delta \approx 1$, where it is possible to completely suppress or substantially reduce vibration near $x_{p}$. As an illustration, consider string vibrations at $n=10, \varphi=0.25, \delta=0.9$. The corresponding dependency of the normalized vibration amplitude $A_{1}$ on $x / l$ in the absence of cross-section modulation $(\chi=0)$ is shown in Figure 1(a), where dashed line corresponds to the analytical solution of equation (9), and solid line to numerical solution (Wolfram Mathematica, NDSolve). Now employ (31), and determine the value of modulation amplitude $\chi$ that will minimize $A_{1}$ near the string end $\left(x_{p}=l\right)$. This gives $\chi=0.26$, and the corresponding dependency of $A_{1}$ on $x / l$ as shown in Figure 1(b). As appears, vibration is completely suppressed near $x_{p}=l$, and is substantially reduced in the whole string. Dashed and solid lines almost coincide in Figures 1 (a)-(b), demonstrating good agreement between numerical and analytical solutions. This agreement is present for a wide range of system parameters tested, so further only the dependencies obtained analytically will be presented.

Considering a non-resonant case, e.g. $n=3, \varphi=2.5, \delta=0.95$, and minimizing vibration near point $x_{p}=0$, one obtains $\chi=0.5$, with corresponding dependencies as shown in Figure 1(c); here solid line corresponds to $\chi=0$, and dashed line to $\chi=0.5$. Another example is shown in Figure 1 (d), where vibration is minimized near the middle of the string. Note that since $k=2 \pi n / l$ the value of string length $l$ does not affect $A_{1}$ and the dependencies presented in Figure 1. 

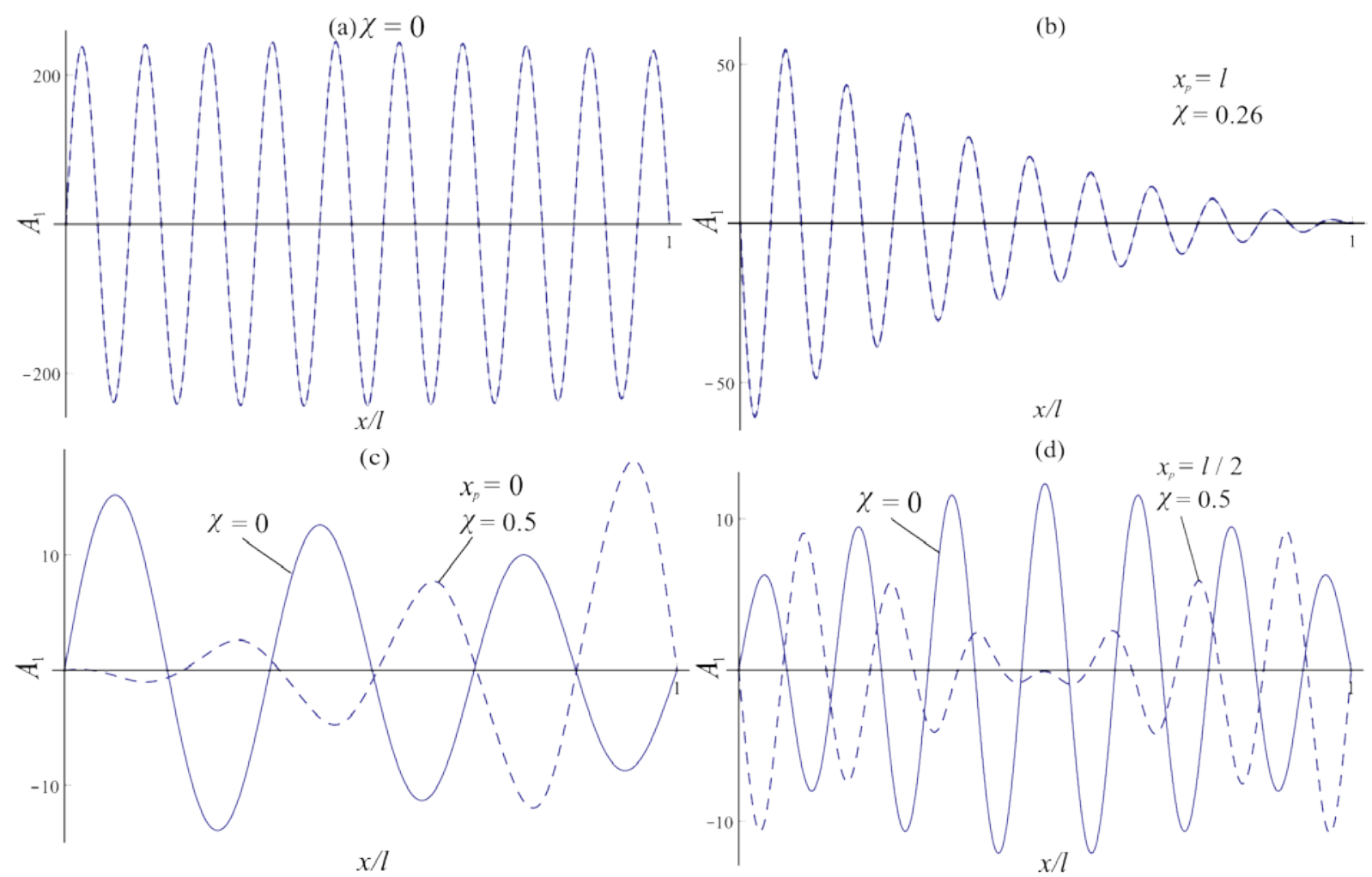

Figure 1. Axial variation of $A_{1}$ for (a)-(b) $n=10, \varphi=0.25, \delta=0.9$;

(c) $n=3, \varphi=2.5, \delta=0.95$; (d) $n=7, \varphi=0, \delta=0.8$.

The proposed technique of vibration suppression turns out to be effective with a broad range of external excitation parameters $\varphi$ and $n$. As an illustration, Figure 2(a) shows the dependency of the ratio $\left.B_{1}\right|_{x=x_{p}} /\left.B_{10}\right|_{x=x_{p}}$ on the phase $\varphi$ for $n=10, \delta=0.9$, and $x_{p}=l$; here $\left.B_{1}\right|_{x=x_{p}}$ is the minimized value of the string vibration amplitude at point $x_{p}$, and $\left.B_{10}\right|_{x=x_{p}}$ is value of this amplitude at $\chi=0$. As is seen, vibration in the prescribed part of the string is less than $5 \%$ of its value without cross-section modulation for almost all $\varphi$. The optimal modulation amplitude $\chi$ strongly depends on $\varphi$, e.g. $\varphi=0.25$ gives $\chi=0.26$, whereas $\varphi=1.25$ gives $\chi=0.99$. Figure 2(b) shows the dependency of the amplitude ratio on the number $n$ of the external excitation harmonic for $\varphi=2.5, \delta=0.95$, and $x_{p}=0$. As appears, vibrations can be substantially reduced for all harmonics.

The effectiveness of the proposed technique of vibration suppression varies with $x_{p}$. There may be regions in which vibrations can be completely suppressed, and regions in which it can be reduced only by $50 \%$. As an illustration, Figure 2(c) shows the dependency of the amplitude ratio on $x_{p}$ when $n=7, \delta=0.8$, and $\varphi=0$. As is seen, vibration near the string ends can be reduced only by $50 \%$, whereas in the middle of the string it can be completely suppressed. This is due to the fact that vibration amplitudes near the string ends in the absence of cross-section modulation are comparatively low, and thus the value of $\left.B_{10}\right|_{x=x_{p}}$ is small. Also, at large modulation amplitudes, e.g. $\chi=0.95$, vibrations turns out to be considerably reduced in the whole string for almost all values of system parameters. 

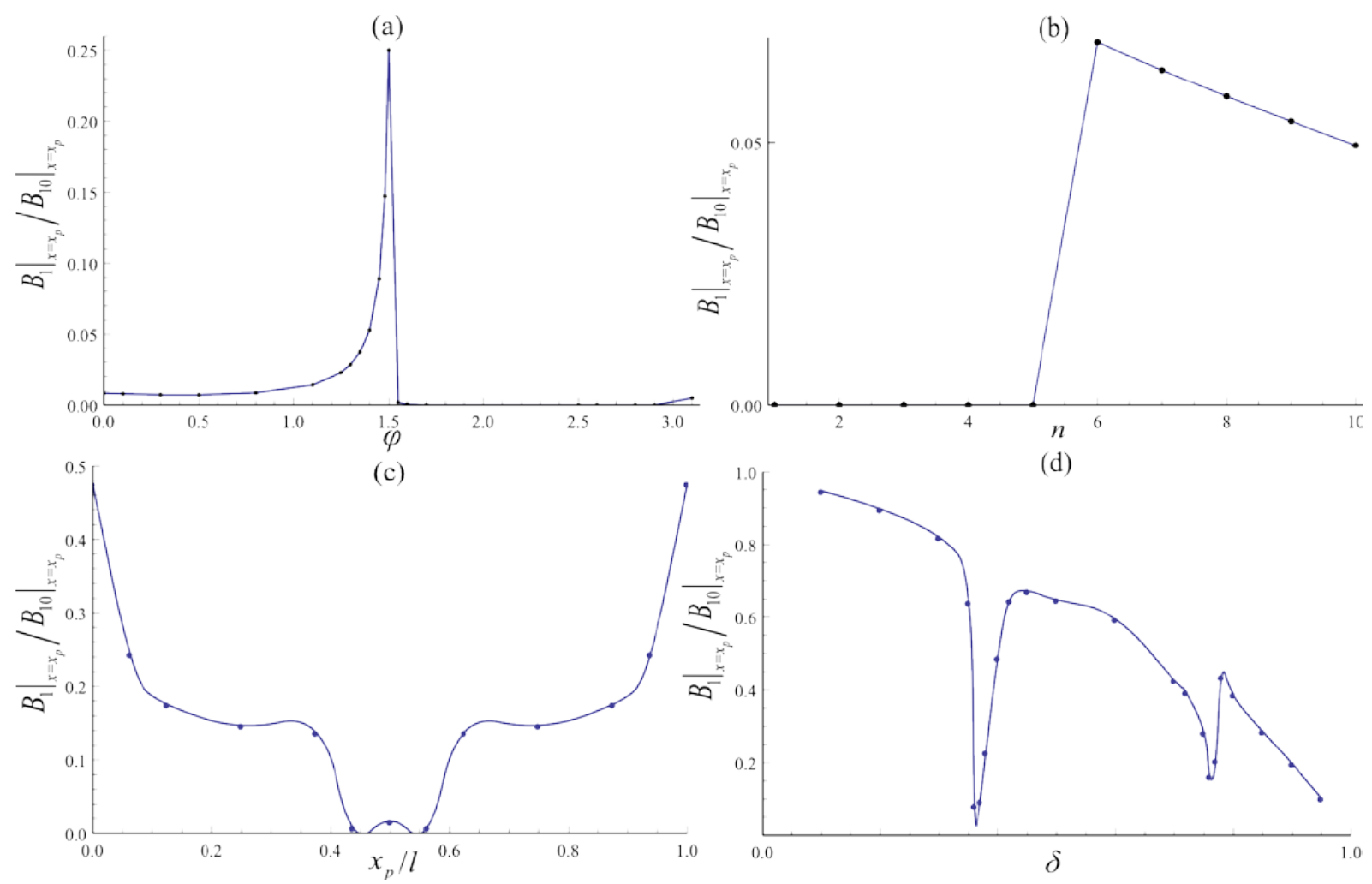

(d)

Figure 2. Dependencies of $\left.B_{1}\right|_{x=x_{p}} /\left.B_{10}\right|_{x=x_{p}}$ on the system parameters: (a) phase $\varphi$ of the external excitation; (b) number $n$ of the external excitation harmonic; (c) point $x_{p}$ near which vibration is minimized; and (d) parameter $\delta$. Here $\left.B_{1}\right|_{x=x_{p}}$ is the minimized value of the string vibration amplitude at point $x_{p}$, and $\left.B_{10}\right|_{x=x_{p}}$ is value of this amplitude when $\chi=0$.

The positive effect of cross-section modulation reduces with decreasing $\delta$, as illustrated by Figure 2(d) for $n=4, \varphi=1.5$, and $x_{p}=l / 3$. When $\delta<<1$, the best way to suppress vibration in any region of the string is to impose the largest possible modulation $(\chi \rightarrow 1)$. However, the effect of the modulation on the system response in this case is much less pronounced than when $\delta \approx 1$.

\section{Spatially uniform external load}

Next we consider the case of spatially uniform external load: $f_{0}(x)=F_{0}$. As an approximation to the sought modulation we employ, similarly to (8):

$$
S(x)=S_{0}\left(1+\chi \cos \left(k_{1} x+\theta_{1}\right)\right),
$$

where $\chi \in[0 ; 1), \theta_{1}$ and $k_{1}$ are unknown parameters to be determined. So, the effect of parametric attenuation of a uniform load is examined and employed here. Note the difference between the case studied in Section 3, where $k$ was the external excitation parameter, and the one considered here, where $k_{1}$ is an optimization parameter. Inserting (32) and $f_{0}(x)$ into (5) one obtains

$$
A_{2}^{\prime \prime}\left(x_{2}\right)+\delta_{1}\left(1+\chi \cos \left(k_{2} x_{2}+\theta_{1}\right)\right) A_{2}\left(x_{2}\right)=1,
$$

where $x_{2}=x / l, \delta_{1}=\omega^{2} \rho S_{0} I^{2} / T, \quad k_{2}=k_{1} l$, and $A_{2}\left(x_{2}\right)=-T A\left(x_{2}\right) / F_{0}$, and now primes denote derivatives with respect to $x_{2}$. The boundary conditions for $A_{2}\left(x_{2}\right)$ are 


$$
A_{2}(0)=A_{2}(1)=0 \text {. }
$$

To simplify the analysis the restriction $\delta_{1}<<k_{2}^{2}$ on the optimization parameter $k_{2}$ is imposed. Other cases, e.g. $\delta_{1} \gg k_{2}^{2}$, can be treated similarly, but the MVA procedure will be more cumbersome.

\subsection{Solution by the method of varying amplitudes}

Employing the MVA for solving equation (33) we search for a solution in the form

$$
A_{2}\left(x_{2}\right)=\alpha\left(x_{2}\right)+B_{11}\left(x_{2}\right) \cos \left(k_{2} x_{2}+\theta_{1}\right)+B_{12}\left(x_{2}\right) \sin \left(k_{2} x_{2}+\theta_{1}\right)+\ldots
$$

This form differs from (11), employed in Section 3, e.g. here the zeroth harmonic is taken into account, making (35) more convenient for solving (33) since constant is present in the right-hand side of this equation. New dependent variables implied in the MVA, e.g. $\alpha$, $B_{11}$ and $B_{12}$ here, or $B_{11}, B_{12}, B_{21}$ and $B_{22}$ in Section 3, are not subjected to any artificial restrictions, e.g. are not required to vary slowly etc. Thus, they can be chosen in any appropriate manner complying the general rules of shifting of variables when solving ordinary differential equations [30].

Taking into account only the written terms in series (35) we obtain the following equations for $\alpha\left(x_{2}\right), B_{11}\left(x_{2}\right), B_{12}\left(x_{2}\right)$ :

$$
\begin{aligned}
\alpha^{\prime \prime} & +\delta_{1}\left(\alpha+\frac{\chi}{2} B_{11}\right)=1, \\
B_{11}^{\prime \prime} & +2 k_{2} B_{12}^{\prime}+\left(\delta_{1}-k_{2}^{2}\right) B_{11}=-\delta_{1} \chi \alpha, \\
\left(B_{12}^{\prime \prime}\right. & \left.-2 k_{2} B_{11}^{\prime}+\left(\delta_{1}-k_{2}^{2}\right) B_{12}\right) \sin \left(k_{2} x_{2}+\theta_{1}\right) \\
& =-\frac{\delta_{1} \chi}{2}\left(B_{11} \cos \left(2\left(k_{2} x_{2}+\theta_{1}\right)\right)+B_{12} \sin \left(2\left(k_{2} x_{2}+\theta_{1}\right)\right)\right) .
\end{aligned}
$$

The right-hand side of (38) will be small in comparison with the leading terms of (37)-(38) if $\left|\delta_{1}-k_{2}^{2}\right|>>\delta_{1} \chi / 2$. Here the case $\delta_{1}<<k_{2}^{2}$ is considered, so this condition is fulfilled. Thus (38) can be approximated by:

$$
B_{12}^{\prime \prime}-2 k_{2} B_{11}^{\prime}+\left(\delta_{1}-k_{2}^{2}\right) B_{12}=0 \text {. }
$$

A particular solution of equations (36), (37), (39) has the form:

$$
\alpha_{0}=\frac{2\left(k_{2}^{2}-\delta_{1}\right)}{\delta_{1}\left(2 k_{2}^{2}-2 \delta_{1}+\delta_{1} \chi^{2}\right)}, \quad B_{110}=\frac{2 \chi}{2 k_{2}^{2}+\delta_{1}\left(\chi^{2}-2\right)}, \quad B_{120}=0
$$

Next we determine the lowest two eigenvalues corresponding to the homogeneous part of (36), (37), (39). From (37):

$$
B_{12}^{\prime}=-\frac{1}{2 k_{2}}\left(\delta_{1} \chi \alpha+\left(\delta_{1}-k_{2}^{2}\right) B_{11}+B_{11}^{\prime \prime}\right)
$$

Differentiating (39) with respect to $x_{2}$ and employing (41) gives:

$$
B_{11}^{\prime \prime \prime}+\delta_{1} \chi \alpha^{\prime \prime}+2\left(\delta_{1}+k_{2}^{2}\right) B_{11}^{\prime \prime}+\left(\delta_{1}-k_{2}^{2}\right)\left(\delta_{1} \chi \alpha+\left(\delta_{1}-k_{2}^{2}\right) B_{11}\right)=0
$$

From (36) we have:

$$
B_{11}=\frac{2}{\chi \delta_{1}}\left(1-\alpha^{\prime \prime}\right)-\frac{2}{\chi} \alpha,
$$

which substituted into (42) gives:

$$
\alpha^{(6)}+\left(2 k_{2}^{2}+3 \delta_{1}\right) \alpha^{\prime \prime \prime}-\frac{\left(\delta_{1}^{2}\left(\chi^{2}-6\right)-2 k_{2}^{4}\right)}{2} \alpha^{\prime \prime}-\frac{\delta_{1}}{2}\left(\delta_{1}-k_{2}^{2}\right)\left(2 k_{2}^{2}+\delta_{1}\left(\chi^{2}-2\right)\right) \alpha=\left(k_{2}^{2}-\delta_{1}\right)^{2}
$$


Consequently, six eigenvalues of (44) are determined; the lengthy expressions for them are not given here. The resulting relations for $\alpha, B_{11}$ and $B_{12}$ are:

$$
\begin{aligned}
& \alpha=\frac{2\left(k_{2}^{2}-\delta_{1}\right)}{\delta_{1}\left(2 k_{2}^{2}-2 \delta_{1}+\delta_{1} \chi^{2}\right)}+C_{1} \cosh \lambda x_{2}+C_{2} \sinh \lambda x_{2}, \\
& B_{11}=\frac{2 \chi}{2 k_{2}^{2}+\delta_{1}\left(\chi^{2}-2\right)}-\frac{2\left(\delta_{1}+\lambda^{2}\right)}{\chi \delta_{1}}\left(C_{1} \cosh \lambda x_{2}+C_{2} \sinh \lambda x_{2}\right), \\
& B_{12}=-\frac{2 k_{2}^{2}\left(\delta_{1}+\lambda^{2}\right)-2\left(\delta_{1}+\lambda^{2}\right)^{2}+\delta_{1}^{2} \chi^{2}}{2 k_{2} \delta_{1} \chi \lambda}\left(C_{2} \cosh \lambda x_{2}+C_{1} \sinh \lambda x_{2}\right) .
\end{aligned}
$$

where $\lambda$ is the lowest eigenvalue of (44). To determine constants $C_{1}, C_{2}$ we employ the boundary conditions (34) for $A_{2}$. Thus, the solution of (33) is composed in the form (35), where $\alpha, B_{11}$, and $B_{12}$ are defined by (45).

\subsection{Vibration suppression in predefined regions of the string}

Here the case of uniformly distributed external load is considered, and component $\alpha\left(x_{2}\right)$ is present in the solution (35), so, instead of minimization criterion (31) the following one will be employed:

$$
\left.A_{2}\right|_{x=x_{p}} \rightarrow \min .
$$

This criterion is a "direct" one; it is chosen due to the fact that, by contrast to Section $3, \lambda$ here is of the same order as $k_{2}$ for a broad range of system parameters, so the amplitudes in (35) are not slowly varying in comparison with $\cos \left(k_{2} x_{2}+\theta_{1}\right)$ and $\sin \left(k_{2} x_{2}+\theta_{1}\right)$. The fulfilment of (46) ensures that vibration near a certain point $x_{p}$ of the string is maximally suppressed. The size of the region in which vibration is minimized depends on the rate of the system's response variation with respect to the spatial coordinate $x$, i.e. on values of the parameters $\lambda$ and $k_{2}$. We note that, due to the boundary conditions, criterion (46) is of no use when $x_{p}=l$ or $x_{p}=0$, so in order to minimize vibration near the string ends it should be applied for $x_{p}$ close to 0 or $l$, e.g. $x_{p}=l / 20$ or $x_{p}=19 l / 20$.

When $\delta_{1}$ is comparatively small $\sqrt{\delta_{1}}<2 \pi$, the proposed technique of vibration suppression allows for considerable vibration reduction in the prescribed part of string. As an illustration, consider the case $\delta_{1}=8$ and suppressing vibration near $x_{p}=l / 2$. Using (46) the optimal parameter values become $k_{2}=5, \quad \chi \rightarrow 1$, and $\theta_{1}=0.6$. The corresponding dependencies of $A_{2}$ on $x_{2}$ are shown in Figure 3(a), where dashed lines represent the analytical solution of equation (33), and solid lines a numerical solution (Wolfram Mathematica, NDSolve). Note that for comparatively small $\delta_{1}$, vibration in any predefined region of the string will be maximally suppressed when the largest possible modulation is imposed $(\chi \rightarrow 1)$. The optimal values of parameters $k_{2}$ and $\theta_{1}$, however, depend strongly on $\delta_{1}$ and $x_{p}$.

For larger $\delta_{1}$ a multitude of parameter values $\chi, k_{2}$ and $\theta_{1}$ exist that enables complete suppression of vibration at the predefined point $x_{p}$ of the string. However, the size of the region near this point, in which vibration is minimized, reduces with increasing $\delta_{1}$ (since $\lambda$ and $k_{2}$ become larger). Considering the example case $\delta_{1}=100$ and minimizing 
vibration near the string end $x_{p}=l$, one finds $k_{2}=23, \chi=0.8$, and $\theta_{1}=2$. The corresponding dependencies of $A_{2}$ on $x_{2}$ in the absence $(\chi=0)$ and presence $(\chi=0.8)$ of cross-section modulation are shown in Figure 3(b). When $\delta_{1}$ is very large $\left(\sqrt{\delta_{1}}>10 \pi\right)$, the proposed technique of vibration suppression becomes less efficient, since the region near $x_{p}$ in which vibration is minimized becomes very narrow; see, e.g. Figure 3(c), where the dependencies of $A_{2}$ on $x_{2}$ are presented for $\delta_{1}=1100$ in the absence (solid line) and presence (dashed line) of cross-section modulation with parameters optimal for $x_{p}=l / 2$.

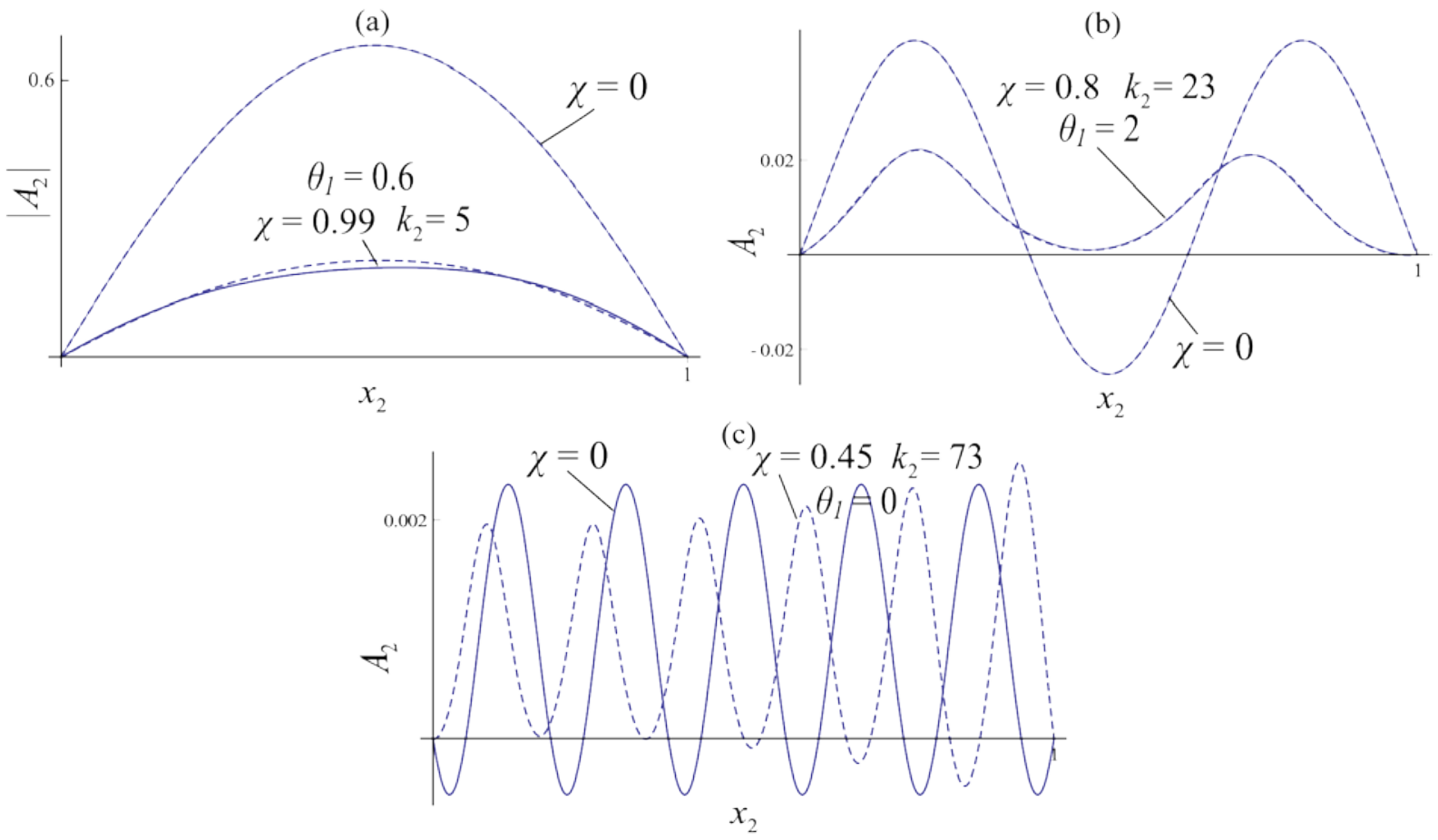

Figure 3. Dependencies of $A_{2}$ on $x_{2}$ for (a) $\delta_{1}=8, x_{p}=l / 2$; (b) $\delta_{1}=100, x_{p}=l$;

$$
\text { (c) } \delta_{1}=1100, x_{p}=l / 2 \text {. }
$$

\section{Arbitrarily distributed external load.}

Finally we consider the case of arbitrarily distributed external load. In Section 2 it was proposed to expand the load distribution function $f_{0}(x)$ in a trigonometric Fourier series (6), and approximate the sought cross-section modulation as the one corresponding to the dominating spatial harmonic, or as a certain superposition of the modulations. In order to reveal the optimal way of introducing modulation, consider a simplified case where $f_{0}$ is a sum of a constant and two harmonics:

$$
f_{0}(x)=F_{01} \cos \left(\tilde{k}_{1} x+\varphi_{1}\right)+F_{02} \cos \left(\tilde{k}_{2} x+\varphi_{2}\right)+F_{03} .
$$

Studying this simplified case we will obtain results which will be valid for any distribution of the external load. The corresponding equation for $A(x)$ takes the form:

$$
A^{\prime \prime}+\delta_{2} \mu(x) A=\sigma_{1} \cos \left(\tilde{k}_{1} x+\varphi_{1}\right)+\sigma_{2} \cos \left(\tilde{k}_{2} x+\varphi_{2}\right)+\sigma_{3},
$$

where $\delta_{2}=\omega^{2} \rho S_{0} / T, \sigma_{p}=-F_{o p} / T, p=1,2,3, \mu(x)$ describes modulation of the crosssection, and now primes denote derivatives with respect to $x$. In Section 3 it was found that the modulation optimal for maximum suppression of a harmonic load $F_{0} \cos (k x+\varphi)$ is 
$S_{0}(1-\chi \cos (2(k x+\varphi)))$. Taking into account these results, as well as those of Section 4, we examine the effect of modulation introduced in various manners defined by:

$$
\mu(x)=1-\chi_{1} \cos \left(2\left(\tilde{k}_{1} x+\varphi_{1}\right)\right)-\chi_{2} \cos \left(2\left(\tilde{k}_{2} x+\varphi_{2}\right)\right)+\chi_{3} \cos \left(k_{3} x+\theta_{3}\right),
$$

where $\chi_{1}, \chi_{2}, \chi_{3}$ take either optimal values, determined in Sections 3-4, or equal zero. Values of parameters $k_{3}, \theta_{3}$ provide maximum suppression with a spatially uniform load $\sigma_{3}$. The following restriction is added to prevent vanishing of the string cross-sectional area: if $S(x)<c_{\min } S_{0}$, then $S(x)=c_{\min } S_{0}$, where $c_{\min }$ determines the minimal possible value of the cross-sectional area.

Numerical experiments show that cross-section modulation corresponding to one of the involved spatial harmonics is more effective than a superposition of the modulations, that is, in most cases modulations reduce the positive effect of each other. As an illustration, consider sting vibrations under action of a spatially harmonic plus uniform load with $\sigma_{1}=\sigma_{3}=0.1 \mathrm{~cm}^{-1}, \sigma_{2}=0$, and $\delta_{2}=1.5 \mathrm{~cm}^{-2}, l=5 \mathrm{~cm}, \quad c_{\min }=0.01, k_{1}=2 \pi / l \mathrm{~cm}^{-1}$, $\varphi_{1}=3$. Vibration is minimized near point $x_{p}=0$. Employing criterion (31), the optimal modulation corresponding to spatially harmonic load has $\chi_{1}=0.29$. Using (46) then gives $k_{3}=2.2 \mathrm{~cm}^{-1}, \theta_{3}=3.3$, and $\chi_{3}=0.99$. The corresponding dependencies of $A$ on $x$ are shown in Figure 4(a). As appears, the most effective modulation is the one corresponding to the spatially harmonic load. Note that $\delta=\delta_{2} / k_{1}^{2} \approx 1$ for this harmonic; here $\delta$ has the same meaning as in Section 3. It turns out that the modulation corresponding to spatial harmonic for which $\delta \approx 1$ will always be the most effective one (see also Section 3). So, if such a harmonic is present in the external excitation, then a modulation corresponding to this harmonic should be imposed. Note that this result is valid for any distribution of the external load.

If there is no such harmonics, or they are small in comparison with the uniform load, then modulation corresponding to the uniform load should be imposed. As an illustration, consider string vibrations at the same values of system parameters as above, but under action of a different spatially harmonic load: $k_{1}=4 \pi / l \mathrm{~cm}^{-1}, \varphi_{1}=0$. For $x_{p}=l / 2$ optimal modulation parameters are: $\chi_{1}=0.99, k_{3}=2.4 \mathrm{~cm}^{-1}, \theta_{3}=3.4$, and $\chi_{3}=0.99$. The corresponding dependencies of $A$ on $x$ are shown in Figure 4(b). As is seen, in this case the modulation corresponding to the spatially uniform load is the most effective one; it considerably suppresses vibration in the prescribed part of the string.

If only spatial harmonics for which $\delta \neq 1$ are present in the external excitation, then modulation corresponding to the one with the largest $\delta$ should be imposed (note that only the case $\delta<1$ is considered here). As an illustration, in Figure 4(c) the dependencies of $A$ on $x$ are presented for a string under action of two spatially harmonic loads: $\sigma_{1}=\sigma_{2}=0.1 \mathrm{~cm}^{-1}$, $\sigma_{3}=0$, and with $\delta_{1}=0.45 \mathrm{~cm}^{-2}, k_{1}=2 \pi / l_{\mathrm{cm}^{-1}}, \varphi_{1}=0, k_{2}=4 \pi / l \mathrm{~cm}^{-1}, \varphi_{2}=2$. Optimal for all $x_{p}$ modulation amplitudes in this case become $\chi_{1}=0.99, \chi_{2}=0.99$.

The abovementioned numerical experiments show that almost in all cases the modulation considerably suppresses vibration in prescribed region of the string. So, the proposed technique of vibration suppression turns out to be effective also in the case of arbitrarily distributed external load. 


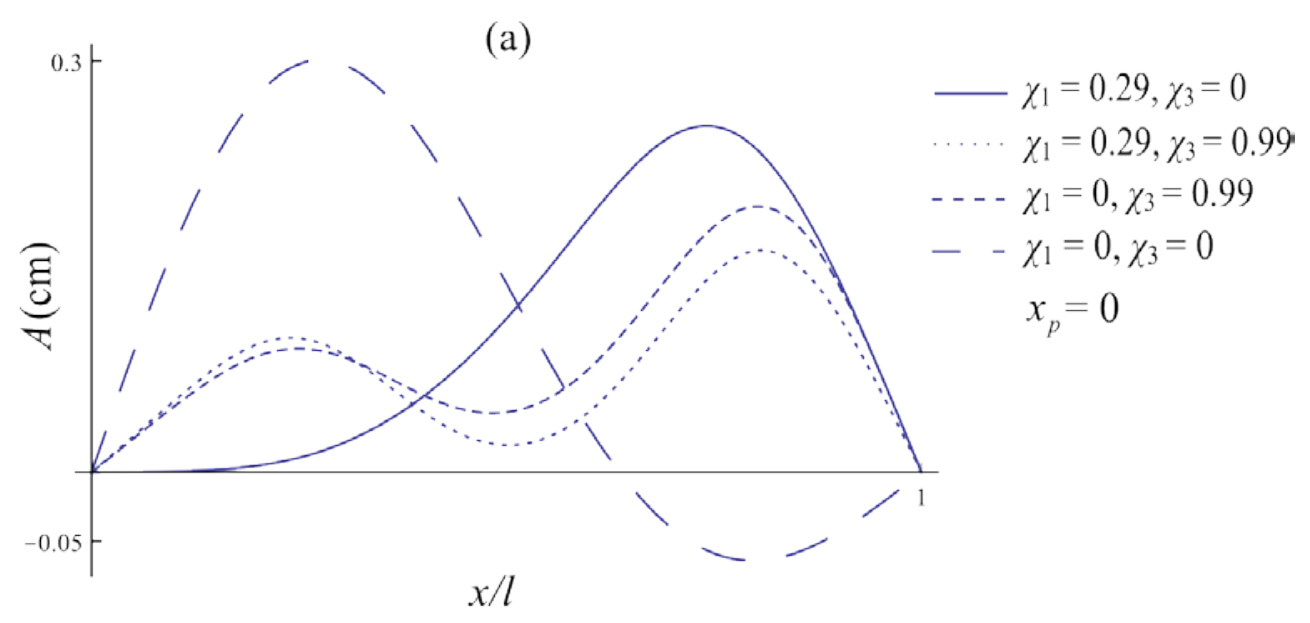

(b)

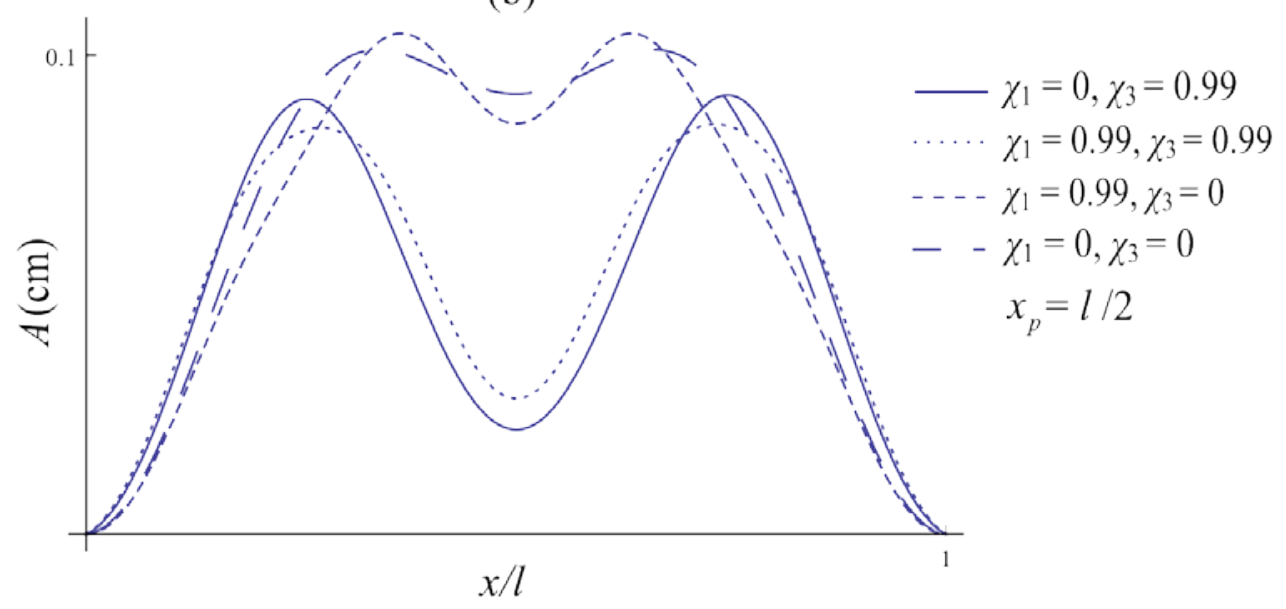

(c)

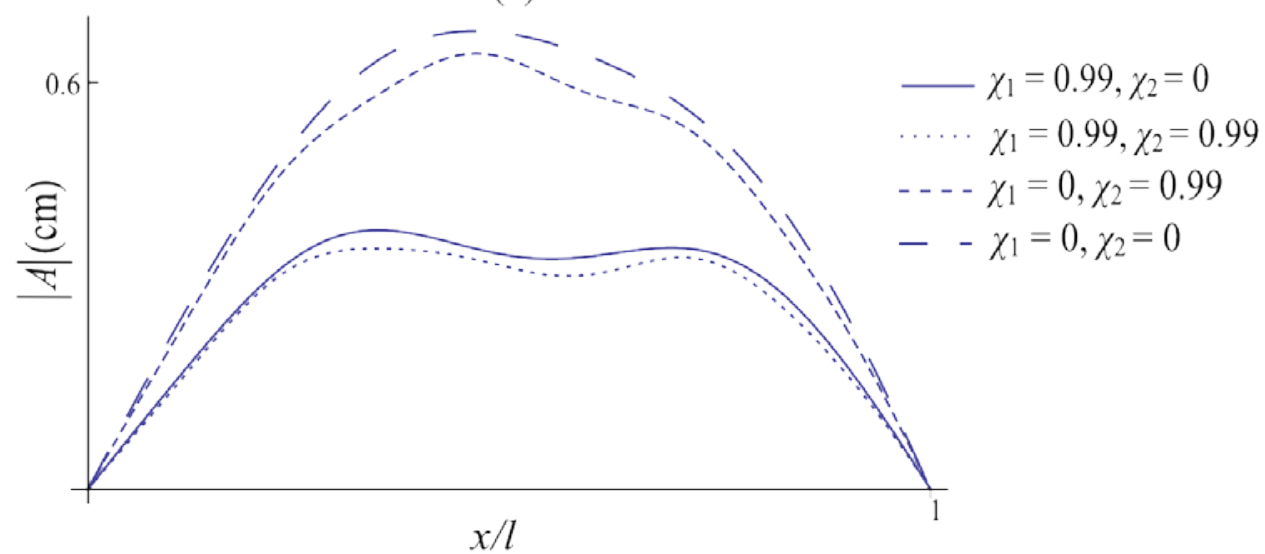

Figure 4. Dependencies of $A$ on $x$ for $l=5 \mathrm{~cm}, C_{\min }=0.01$ and values of other parameters specified in the text.

\section{Conclusions}

A problem of vibration suppression in a bounded structure subjected to action of an external distributed load by using continuous spatial modulations of its parameters is considered. As an example, stationary vibrations of a string under action of a distributed time-periodic force are studied. This system in a simplified form models such processes as interaction between membranes and colloids, oscillations of transmission lines under action of rain and wind, and dynamics of suspension bridges and stay cables. Suppression of vibration in predefined regions of the string is carried out by continuous spatial modulation of its cross-section. 
A novel approach, named the method of varying amplitudes, is employed in the analysis. This approach is applicable for solving differential equations without a small parameter, and may be considered as a natural continuation of the classical methods of harmonic balance and averaging. As a result, optimal parameters for the string cross-sectional area modulation are determined for the cases of harmonically, uniformly and arbitrarily distributed load, which allows for completely suppressing or considerably reducing vibration in the prescribed part of the string (compared to the case without modulation). Conditions where the proposed technique of vibration suppression is most effective are determined. The analytical results are validated by a series of numerical experiments, in all cases showing good agreement. Extension of the proposed technique of vibration suppression to cases of temporally random external load is reserved for future work.

\section{Acknowledgments}

The work is carried out with financial support from the Danish Council for Independent Research and FP7 Marie Curie Actions - COFUND: DFF - 1337-00026.

\section{References}

[1] Margolis, D.L., 1983. A procedure for comparing passive, active, and semi-active approaches to vibration isolation. Journal of the Franklin Institute, 315 (4), pp. 225-238.

[2] Kravchun, P.N., Prudnikov, E.V., Chernyshev, K.V., 1986. Optimization of the length of the connecting wave-guides in one-dimensional sound and vibration isolators based on periodic structures. Soviet Physics Acoustics - USSR, 32 (4), pp. 344-347.

[3] Lurie, K.A., 1997. Effective properties of smart elastic laminates and the screening phenomenon. Int. J. Solids Structures, 34 (13), pp. 1633-1643.

[4] Braun, S., Ewins, D. J., Rao, S. S., et al., 2002. Encyclopedia of Vibration, Academic Press.

[5] Bendsøe, M.P., Sigmund, O., 2003. Topology Optimization - Theory, Methods and Applications, Springer-Verlag.

[6] Virgin, L.N., Santillan, S.T., Plaut, R.H., 2008. Vibration isolation using extreme geometric nonlinearity. Journal of Sound and Vibration, 315 (3), pp. 721-731.

[7] Ibrahim, R.A., 2008. Recent advances in nonlinear passive vibration isolators. Journal of Sound and Vibration, 314, pp. 371-452.

[8] Hansen, C., 2013. Active Control of Noise and Vibration, CRC Press.

[9] Krylov, V., Sorokin, S., 1997. Dynamics of elastic beams with controlled distributed stiffness parameters. Smart Materials and Structures, 6, pp. 573-582.

[10] Lurie, K.A., 2006. An Introduction to Mathematical Theory of Dynamic Materials, Springer-Verlag.

[11] Blekhman, I.I., 2008. Vibrational dynamic materials and composites. Journal of Sound and Vibration, 317, pp. 657-663.

[12] Jensen, J.S., 2009. Space-time topology optimization for one-dimensional wave propagation. Computer Methods in Applied Mechanics and Engineering, 198, pp. 705-715.

[13] Mead, D.J., 1973. A general theory of harmonic wave propagation in linear periodic systems with multiple coupling. Journal of Sound and Vibration, 27, pp. 235-260.

[14] Mead, D.J., 1996. Wave propagation in continuous periodic structures: research contributions from Southampton. Journal of Sound and Vibration,190 (3), pp. 495-524.

[15] Bakhvalov, N.S., Panasenko, G.P., 1989. Homogenization: Averaging Processes in Periodic Media, Kluwer.

[16] Mace, B.R., Duhamel, D., Brennan, M.J., Hinke, L., 2005. Finite element prediction of wave motion in structural waveguides. Journal of the Acoustical Society of America, 117, pp. 2835-2843. 
[17] Soe-Knudsen, A., Sorokin, S., 2011. On accuracy of the wave finite element predictions of wavenumbers and power flow: a benchmark problem. Journal of Sound and Vibration, 330, pp. 2694-2700.

[18] Mahmoudia, M., et al., 2014. Interaction of stable colloidal nanoparticles with cellular membranes. Biotechnology Advances, 32, pp. 679-692.

[19] Hagedorn, P., 1987. Wind-excited vibrations of transmission lines: A comparison of different mathematical models. Mathematical Modelling, 8, pp. 352-358.

[20] Burton, D., Cao, D.Q., Tucker, R.W., Wang C., 2005. On the stability of stay cables under light wind and rain conditions. Journal of Sound and Vibration, 279, pp. 89-117.

[21] Blekhman, I.I., 2000. Vibrational Mechanics, World Scientific.

[22] Blekhman, I.I., 2004. Selected Topics in Vibrational Mechanics, World Scientific.

[23] Magnus, K., 1965. Vibrations, Blackie.

[24] Bogoliubov, N.N., Mitropolskii, Ju.A., 1961. Asymptotic Methods in the Theory of Nonlinear Oscillations, Gordon and Breach.

[25] Sanders, J.A., Verhulst, F., 1985. Averaging Methods in Nonlinear Dynamical Systems, Springer-Verlag.

[26] Nayfeh, A.H., Mook, D.T., 1979. Nonlinear Oscillations, Wiley-Interscience.

[27] Yakubovich, V.A., Starzhinskii, V.M., 1975. Linear Differential Equations with Periodic Coefficients, John Wiley \& Sons.

[28] Sorokin, V.S., 2013. A new approach to the analysis of oscillations of one-dimensional spatially periodic structures. Journal of Sound and Vibration, 332 (14), pp. 3552-3563.

[29] Sorokin, V.S., 2014. Analysis of motion of inverted pendulum with vibrating suspension axis at low-frequency excitation as an illustration of a new approach for solving equations without explicit small parameter. International Journal of Non-Linear Mechanics, 63, pp. 19.

[30] Arnol'd, V.I., 1992. Ordinary Differential Equations, Springer. 